\title{
ADESÃO E REAÇÕES DE USUÁRIOS AO TRATAMENTO DA MALÁRIA: IMPLICAÇÕES PARA A EDUCAÇÃO EM SAÚDE
}

\author{
Annelita Almeida Oliveira Reiners ${ }^{1}$, Rosemeiry Capriata de Souza Azevedo ${ }^{2}$, Hebert Almeida Ricci ${ }^{3}$ Taísa \\ Guimarães de Souza ${ }^{4}$
}

\footnotetext{
${ }^{1}$ Doutora em Enfermagem. Professora da Faculdade de Enfermagem da Universidade Federal de Mato Grosso (UFMT). Mato Grosso, Brasil. E-mail: reiners17@hotmail.com

${ }^{2}$ Doutora em Enfermagem. Professora da Faculdade de Enfermagem da UFMT. Mato Grosso, Brasil. E-mail: capriata@terra. com.br

${ }^{3}$ Aluno de graduação da Faculdade de Enfermagem da UFMT. Bolsista PIBIC/CNPq. Mato Grosso, Brasil. E-mail: hebertricci@ yahoo.com.br

${ }^{4}$ Aluno de graduação da Faculdade de Enfermagem da UFMT. Bolsista PIBIC/CNPq. Mato Grosso, Brasil. E-mail: taisa_guima@ hotmail.com
}

RESUMO: Estudo exploratório-descritivo cujo objetivo foi descrever a adesão e reações de usuários em relação ao tratamento antimalárico em um município de Mato Grosso, Brasil. Os dados foram coletados com 16 usuários através de entrevistas semi-estruturadas e analisados usando-se a Análise de Conteúdo. O estudo revelou que há pessoas que aderem parcialmente ao tratamento antimalárico e outras que procuram tratamentos alternativos. Aos primeiros sinais e sintomas da malária, a maior parte dos usuários procura os serviços de saúde para diagnóstico e tratamento, provavelmente por ter conhecimento sobre a doença, a transmissão e o tratamento da mesma; pela facilidade de acesso ao serviço que oferece diagnóstico e tratamento gratuito; e pelo bom relacionamento com os agentes de saúde. Concluiu-se que apesar do sucesso de algumas ações do governo para controle da malária, ainda há necessidade de investimento em ações educativas e de vigilância em saúde.

DESCRITORES: Conduta de saúde. Cooperação do paciente. Educação em saúde. Malária.

\section{USER ADHERENCE AND REACTIONS TO MALARIA TREATMENT: IMPLICATIONS FOR HEALTH EDUCATION}

\begin{abstract}
This descriptive and exploratory study proposed to describe the user adherence and their reactions to anti-malaria treatment in a municipality in the western region of Brazil. Data was collected through interviewing 16 users and analyzed using Content Analysis. The study revealed that there are people who adhere partially to anti-malaria treatment and others who seek alternative treatments. The results showed that upon the first signs and symptoms starting, most users sought health care services for diagnosis and treatment, probably from knowledge about the disease, transmission, and treatment. Justifications given for such decision were for the ease of access the public health service, which offers free diagnosis and treatment, and the good rapport with the health care workers. It was concluded that although some government actions to control malaria have seen success there is still need for investment in health education actions and health surveillance.
\end{abstract}

DESCRIPTORS: Health behavior. Patient compliance. Health education. Malaria.

\section{ADHESIÓN Y REACCIONES DE LOS USUARIOS AL TRATAMIENTO DE LA MALARIA: IMPLICACIONES PARA LA EDUCACIÓN EN SALUD}

RESUMEN: Se trata de un estudio exploratorio descriptivo con el objetivo de describir la adhesión y las reacciones de los usuarios en relación al tratamiento contra la malaria en un municipio de Mato Grosso, Brasil. La recolección de los datos se hizo por medio de entrevistas semiestructuradas a 16 usuarios, y se analizaron utilizando el Análisis de Contenido. El estudio reveló que hay personas que adhieren en parte al tratamiento de la malaria y otras que buscan tratamientos alternativos. Los resultados mostraron que a los primeros signos y síntomas de la malaria, la mayoría de los usuarios buscan los servicios de salud, probablemente por tener conocimiento sobre la enfermedad, su transmisión y tratamiento; por la facilidad de acceso a la salud pública con diagnóstico y tratamiento gratuito, y por la buena relación con los trabajadores de la salud. Se concluyó que a pesar del éxito de algunas acciones del gobierno para controlar la malaria, hay necesidad de invertir en educación y vigilancia de la salud.

DESCRIPTORES: Conducta de salud. Cooperación del paciente. Educación en salud. Malaria. 


\section{INTRODUÇÃO}

A malária é um grave problema de saúde pública devido a sua alta incidência e às consequências que trazem às pessoas acometidas pela doença, influenciando significativamente o potencial de desenvolvimento de países, regiões e estados, pelos múltiplos custos que acarreta ${ }^{1}$. Incide principalmente nas áreas tropicais e subtropicais do planeta e, mesmo nessas regiões, sua distribuição não é homogênea. Ocorre principalmente na África, no Sudeste Asiático e na Região Amazônica da América do Sul, sendo que sua incidência maior é no continente africano, ao sul do deserto de Saara, onde ocorrem $80 \%$ do total de casos e mortes por malária do mundo, principalmente de crianças jovens. ${ }^{2}$

No Brasil, a doença incide predominantemente na chamada Amazônia Legal, que compreende os Estados do Acre, Amapá, Amazonas, Maranhão, Mato Grosso, Pará, Rondônia, Roraima e Tocantins, prevalecendo as infecções pelo Plasmodium vivax e pelo Plasmodium falciparum, principalmente em populações vivendo em condições insatisfatórias de habitação e trabalho. Nessas populações, a ocorrência da malária está relacionada à ocupação desordenada de terras, à exploração manual de minérios, a projetos de assentamento e colonização agrária e à intensa migração de pessoas da zona rural para a periferia de cidades amazônicas. ${ }^{3}$

Nessa região, o risco de transmissão é alto, com áreas apresentando Incidência Parasitária Anual (IPA) - número de exames positivos de malária por mil habitantes - superior a 100 casos por 1.000 habitantes, embora nos Estados de Rondônia, Roraima, Mato Grosso, Maranhão e Tocantins tenha havido queda expressiva no período de 1990 e $2002 .^{4}$

Esse decréscimo é atribuído ao Programa Nacional de Controle de Malária (PNCM), que teve por objetivos melhorar e ampliar o atendimento aos portadores de malária, reduzir a morbi-mortalidade pela doença na região da Amazônia Legal, diminuir o número de ocorrência de formas graves, o número de internações e óbitos, evitar o surgimento de epidemias localizadas, minimizar o problema da transmissão em áreas urbanas e manter a ausência da doença em locais onde a transmissão já não existe. Apesar disso, no período de 2003 a 2005, o índice parasitário da doença voltou a crescer em quase todos os estados, porém não mais atingiu os valores do início da década de $1990 .^{5}$
A despeito da alta endemicidade da doença na região, o diagnóstico e o tratamento dos casos de malária gradualmente vêm sendo incorporados aos serviços de saúde locais ${ }^{6}$ e o desenvolvimento da terapia antimalárica está associado a acentuadas reduções na morbidade e mortalidade.

Entretanto, apesar dos esforços empreendidos por governos e organizações no combate à doença, barreiras para o cuidado e controle da malária são reconhecidas e provêm tanto do sistema e serviços de saúde, quanto do profissional ou do usuário. Deste último, obstáculos ao controle da doença podem vir do seu comportamento em relação ao tratamento.

Na medida em que esses obstáculos existam para impedir que o tratamento seja eficaz, é certo que consequências ocorram tanto para o usuário quanto para a família, o serviço de saúde, a comunidade e o governo. Especificamente em relação à malária, sabe-se que o não cumprimento do tratamento como prescrito afeta a melhora e a cura do usuário, leva ao desenvolvimento de resistência aos medicamentos, aumenta o risco de transmissão da doença, bem como representa uma perda econômica substancial.

Órgãos governamentais e pesquisadores têm recomendado a realização de estudos que levem em conta os aspectos comportamentais e socioculturais das pessoas acometidas pela malária, no sentido de buscar melhorias na atenção à saúde do usuário e dispensação de medicamentos..$^{7-8} \mathrm{Tal}$ recomendação advém do fato de haver necessidade de se preocupar com os aspectos subjetivos envolvidos nos comportamentos das pessoas quando adoecem.

O desenvolvimento dessas pesquisas pode ser visto como uma extensão do trabalho de combate à malária, pois o entendimento das reações e da motivação das pessoas em relação ao tratamento da doença é fundamental para a implementação de estratégias de controle que sejam adequadas a cada realidade, principalmente em relação à educação em saúde, pois ela deve ser realizada levando em consideração as pessoas, como sujeitos e coletividades que optam por viver de acordo com suas escolhas, criando novas possibilidades de satisfação de necessidades, desejos e interesses. ${ }^{9}$

Diante dessas considerações desenvolveu-se a seguinte questão de pesquisa: Qual a adesão e as reações dos usuários em relação ao tratamento antimalárico prescrito?

Este estudo teve por objetivo descrever a adesão e reações de usuários dos serviços públicos 
de saúde de um município de Mato Grosso, Brasil em relação ao tratamento antimalárico preconizado pelo Ministério da Saúde (MS).

Esta pesquisa foi financiada pela Fundação de Amparo à Pesquisa de Mato Grosso (FAPEMAT).

\section{METODOLOGIA}

O estudo foi do tipo descritivo-exploratório de abordagem qualitativa, de forma retrospectiva. Como acontece em estudos de abordagem qualitativa, a amostra dos sujeitos não foi pré-determina$\mathrm{da}$, mas intencionalmente procurou-se por sujeitos que poderiam fornecer as informações necessárias ao alcance do objetivo da pesquisa. Os critérios definidos para a seleção dos sujeitos foram os de que estes deveriam ser usuários da rede básica de saúde, maiores de 18 anos, de ambos os sexos, que declarassem ter contraído malária nos últimos cinco anos. Inicialmente, pediu-se para os agentes de saúde da cidade que indicassem as pessoas que preenchiam os critérios estabelecidos, depois foi solicitada aos participantes a designação de outras pessoas. O tamanho da amostra foi definido usando-se o princípio da saturação dos dados ${ }^{10} \mathrm{ou}$ seja, os sujeitos foram incluídos até o ponto em que não foi obtida nenhuma informação nova e estava ocorrendo redundância dos dados.

A pesquisa foi realizada no município de Juruena, localizado na região noroeste do Estado de Mato Grosso, à 893 km da capital, Cuiabá. O município possui uma área de $3.190 \mathrm{~km}^{2} \mathrm{e}$ uma população de 8.731 habitantes ${ }^{11}$ cuja atividade econômica baseia-se na indústria madeireira que absorve quase toda a mão-de-obra da área urbana. ${ }^{12}$

Juruena é considerada área endêmica da malária. No período de 2003 a 2004 houve um surto importante da doença decorrente da implantação de assentamentos rurais e da atividade garimpeira desenvolvida na região. Nesta época, o IPA atingiu valores entre 41,0 e 116,8/1000 hab., alcançando o primeiro lugar no ranking das doenças transmissíveis no estado. ${ }^{13}$

A rede de serviços de saúde da cidade é pública e composta por um hospital e três unidades básicas de saúde da família, duas urbanas e uma rural. O diagnóstico da malária é rotineiramente realizado pelos agentes de endemias do Setor de Vigilância Ambiental e de Endemias, por meio do exame da gota espessa o qual é simples, eficaz e de baixo custo. Após identificação do plasmódio, esses agentes fornecem gratuitamente aos usuários os antimaláricos - cloroquina, primaquina, artemeter-lumefantrina, artesunato, quinina, clindamicina doxiciclina, mefloquina - de acordo com os esquemas terapêuticos preconizados pelo MS para cada tipo de malária adquirida. ${ }^{14}$

A coleta de dados foi realizada nos meses de agosto e setembro de 2007, após a aprovação do projeto no comitê de ética local sob o protocolo de $n^{\circ}$ 064/2005/CEP/SES-MT, atendendo assim os preceitos éticos legais vigentes na Resolução $n^{\circ}$ 196/96 do Conselho Nacional de Saúde.

As informações foram obtidas por meio de entrevistas semi-estruturadas, utilizando-se um roteiro de entrevista com questões fechadas referentes a dados sociodemográficos dos usuários e perguntas abertas relacionadas ao comportamento do usuário portador de malária ao tratamento antimalárico. As entrevistas foram realizadas pelas pesquisadoras e por alunos previamente capacitados e treinados para tal, após a obtenção do consentimento escrito dos usuários.

Na avaliação da adesão dos usuários ao tratamento da malária foi necessário considerar que uma das limitações deveu-se ao fato de a pesquisa ter sido realizada em um período que não coincidiu com os episódios de malária que os usuários tiveram, fazendo com que se contasse apenas com o autorrelato sem a possibilidade de comparar o que eles diziam com a verificação das cartelas de medicamentos.

O autorrelato, embora possa sofrer os vieses da subjetividade e do esquecimento, ainda é considerado uma forma de medida de adesão que pode produzir bons indicadores, desde que os questionamentos sejam conduzidos de modo não coercitivo e que os pesquisadores garantam total sigilo e respeito aos respondentes..$^{15}$

Nesta pesquisa, a avaliação da adesão dos usuários ao tratamento antimalárico foi feita mediante questionamento direto a eles, utilizando as seguintes perguntas: Você seguiu o tratamento como recomendado? Tomou todos os comprimidos receitados? Sobrou algum medicamento na cartelinha? Tomou os medicamentos nos horários recomendados? Você se esqueceu de tomar os medicamentos? Em algum momento você teve vontade de parar de tomar os medicamentos? A fim de aumentar a acuidade das respostas, ao longo da entrevista essas perguntas foram feitas aos usuários várias vezes e de diferentes formas, atentando para inconsistências ou incoerências em suas declarações, como o relato de ter se esquecido de tomar o medicamento no horário ou de que a malária voltou. 
Quando o usuário declarava ter seguido o tratamento conforme recomendado pelo profissional de saúde e, ao longo da entrevista, não terem sido constatadas inconsistências ou incoerências em suas declarações, considerava-se que provavelmente ele tinha aderido ao tratamento.

De outro modo, quando no relato havia tais inconsistências ou incoerências, considerava-se que provavelmente ele não tinha aderido ao tratamento. Por fim, considerava-se que o usuário não tinha aderido ao tratamento quando ele declarava não ter seguido o tratamento antimalárico preconizado pelo MS.

As entrevistas foram transcritas, lidas várias vezes e os dados codificados e categorizados usando-se a estratégia de análise qualitativa Análise de Conteúdo, na qual o pesquisador busca os significados a partir de um esquema de classificação de códigos correspondentes, os quais são utilizados para distribuir e organizar os dados de forma que as estruturas se conectem às categorias temáticas. ${ }^{10}$ As categorias que emergiram foram: "Reações dos usuários em relação ao tratamento antimalárico" e "Adesão ao tratamento e motivos para aderir". As mesmas foram discutidas utilizando-se o referencial da adesão a tratamentos e da educação em saúde.

\section{RESULTADOS E DISCUSSÃO}

\section{Características da população estudada}

Ao todo, participaram da pesquisa 16 usuários, sendo oito (50\%) do sexo masculino e oito $(50 \%)$ do feminino. A maior parte deles $(93,7 \%)$ morava em um assentamento na área rural do município de Juruena. A faixa etária variou entre 19 e 60 anos. A maioria (87,5\%) era casada, possuía o ensino fundamental incompleto (100\%) e tinha como ocupação principal a agricultura familiar $(93,7 \%)$. Dez $(62,5 \%)$ dos usuários afirmaram ter adquirido malária tanto do tipo falciparum quanto do vivax, enquanto seis $(37,5 \%)$ deles tiveram apenas a do tipo vivax.

\section{Reações dos usuários em relação ao trata- mento antimalárico}

\section{Busca pelo tratamento}

Por meio dos relatos dos usuários, foi possível constatar que eles apresentaram sinais e sintomas típicos da enfermidade como cefaléia, febre e calafrios - [...] dava dor de cabeça, frio, febre (E2) - no entanto, dos 16 usuários pesquisados, somente dois declararam não ter seguido o tratamento para a malária preconizado pelo MS, preferindo o autotratamento com medicamentos homeopáticos e plantas medicinais. Considerando a definição de adesão ao tratamento estabelecida para este estudo, esses usuários, portanto, não aderiram ao tratamento P: Quando a senhora teve malária pela primeira vez, pensou em ir lá no serviço de saúde? E: Olha eu nem fui lá... (E2).

Já os outros 14 usuários pesquisados, tão logo apresentaram os sintomas da malária, buscaram o serviço de saúde para fazer o exame de sangue para o diagnóstico e receber os medicamentos apropriados ao tipo de malária adquirida. [Sentia] dores nas pernas, fraqueza. Daí eu fui à rua [cidade] tirar o sangue, fiz exame e deu malária (E6). Quando a gente via que era malária, já procurava os meninos lá no posto de saúde. Eles passavam aqui, coletavam sangue, faziam o exame, aí a gente tomava os remédios, tudo certinho e loguinho tava boa (E11).

Os usuários desta pesquisa, mesmo fazendo uso de plantas medicinais e de medicamentos antipiréticos como tratamento complementar para amenizar os sintomas da malária, não preteriam o tratamento alopático específico para a doença, buscando-o em primeiro lugar por acreditar que somente os antimaláricos poderiam curá-los. Não. Falar que vai deixar de tomar o medicamento [e substituir] pelo chá não (E16); [...] são os remédios da malária que fazem a gente sarar. Se a gente não tomar todo o remédio não sara. (E7).

Em uma revisão de literatura sobre busca de tratamento para a malária, constatou-se ${ }^{16}$ que geralmente as pessoas esperam, em média, um período de três dias para procurar o tratamento para a doença. A reação dos usuários deste estudo em relação ao tratamento da malária, portanto, mostrou ser diferente. $E$ - Começou a dar moleza no corpo, febre, dor na nuca. Dói assim, aqui na frente, dói tudo. Começa assim. Aíe é só cama mesmo e cobertor. P - Quando o senhor percebeu isso, fez o que?E - Procurei o posto de saúde (E3).

É reconhecido que o controle da malária depende, dentre outros fatores, do comportamento das pessoas em relação às medidas profiláticas, ao diagnóstico e ao tratamento. A busca precoce pelo diagnóstico e o início do tratamento apropriado o mais rápido possível contribui significativamente para prevenir o óbito, o sofrimento humano, eliminar as fontes de infecção e reduzir o uso desnecessário de antimaláricos,, 717 sendo importante na redução do impacto da malária na pessoa, na família e na comunidade. 
É difícil chegar a conclusões definitivas neste estudo, contudo, através da análise dos relatos dos usuários, presume-se que um fator que pode ter cooperado para que eles tivessem esse tipo de comportamento é o fato de que tinham conhecimento de alguns dos sintomas clássicos da malária, geralmente a febre e os calafrios. Do mesmo modo, tinham um entendimento razoável sobre a forma como a doença é transmitida, inclusive fazendo relação com o contexto onde vivem (mato, rio, garimpo, água parada), identificando-o como propício à infecção Eu comecei com febre em casa e a gente já sabia que a malária estava avançada, pois tinha febre alta, tirava até o apetite da gente. Aí dava uma moleza no corpo e aquele trem ruim (E12); P - Mas como a senhora acha que pegou a malária? E - O mosquito da malária sai à noite e cedo. Aqui pertinho tinha os garimpeiros que trabalhavam ali pertinho. Acho que foi de lá, porque antes desse garimpo não tinha malária. P - O que a senhora acha que tinha lá no garimpo que trouxe a malária?E - Água parada. Eu acho que nos buracos que eles trabalhavam ficava água parada. Acho que é daí. Eu estou achando que foi até o pessoal de fora que trouxe essa malária prá cá porque de primeiro não tinha (E5).

Semelhante resultado foi encontrado em alguns estudos, nos quais os autores constataram que os participantes de suas pesquisas tinham um conhecimento considerável dos sinais e sintomas da malária, bem como do modo de transmissão da doença. ${ }^{18-20}$ Ações de educação em saúde, portanto, podem ter contribuído para que os usuários deste estudo tenham buscado o diagnóstico e tratamento precoces. Nessa esfera de atenção, a educação em saúde certamente ajuda no combate à malária. Ainda que não apenas o fornecimento de informações garanta a adesão ao tratamento, é indubitável que quanto mais informada a população estiver sobre as manifestações clínicas da doença, sua gravidade, os fatores determinantes e colaboradores da sua incidência, bem como sobre as medidas disponíveis para redução da influência desses fatores e o tratamento, maior a probabilidade de participar ativamente de seu controle. ${ }^{7}$

É possível que o conhecimento que os usuários tinham sobre a enfermidade e o tratamento esteja relacionado com as informações e instruções sobre a doença, a forma de transmissão e o tratamento fornecidos pelos agentes de saúde do local, intensificadas a partir da ocorrência do surto de malária em 2003/2004. Sempre eles alertam a gente prá fazer tudo certinho(E3). O conhecimento sobre a malária e a educação desempenham um papel importante nas percepções e práticas de controle da doença. ${ }^{21}$
Ao fornecerem conhecimento aos usuários, os agentes estariam preenchendo um dos requisitos básicos da educação em saúde preconizados atualmente, que é fortalecer a capacidade individual e coletiva dos sujeitos de lidar com os múltiplos fatores que contribuem para seu adoecer. ${ }^{22}$

Entretanto, apenas o fornecimento de informações não é suficiente para que o conhecimento produza efeito sobre o comportamento das pessoas em busca de tratamento apropriado. Estudos têm assinalado a escolaridade das pessoas como um dos elementos relacionados à capacidade que elas têm para entender as informações e instruções que lhes são fornecidas. ${ }^{23-24}$

Nesta pesquisa, é provável que o grau de escolaridade dos usuários tenha colaborado com o nível de conhecimento que eles tinham sobre a doença, na medida em que relatavam ter facilidade para entender as informações e instruções. Eu entendia o que eu tinha que fazer. Tinha que tomar os remédios, acompanhar certinho (E16).

Presume-se também que aos fatores que contribuem para a busca precoce pelo diagnóstico e tratamento agrega-se a experiência prévia de alguns dos usuários com a doença, pois, como já foi mencionado, Juruena é área endêmica para a malária.

A vivência anterior com a enfermidade tem sido apontada como um fator significativo no controle da malária, pois contribui para que as pessoas identifiquem os sinais e sintomas e busquem o tratamento específico. ${ }^{24}$

Aliado a esses fatores, o acesso ao serviço de saúde pode ter colaborado para a busca do tratamento oficial pelos usuários. A oferta de tratamento apropriado previne a progressão da malária e a manifestação de formas severas da doença. ${ }^{17}$

No que se refere ao avanço na implementação das ações de controle da malária, Mato Grosso apresenta grande, extensa e regular participação dos municípios nessas atividades e promove a expansão da rede de diagnóstico e tratamento, sendo que o intervalo de tempo entre um e outro é inferior a 24 horas, na maioria dos casos. ${ }^{3}$

$\mathrm{O}$ acesso ao serviço de saúde, no caso de Juruena, contribui em três sentidos. O primeiro pelo fato de o município disponibilizá-lo aos moradores acometidos pela malária por meio das unidades de atenção básica e do hospital, bem como do Setor de Vigilância Ambiental e de Endemias que faz a busca ativa de casos. Segundo, porque o serviço de saúde atende prontamente a pessoa que busca 
o diagnóstico, fazendo o exame de sangue e, em pouco tempo, o resultado está à disposição dela. Por último, devido à disponibilização gratuita dos antimaláricos aos usuários, eliminando o obstáculo financeiro para a aquisição dos medicamentos que é comum em muitos locais onde a malária é endêmica. O pessoal da saúde que veio aqui olhava as pessoas que estavam mal, fazia a lâmina e quem tinha a malária eles iam à cidade e buscavam o remédio. Alguns iam prá lá e pegavam o medicamento (E5).

Por fim, o bom relacionamento entre os agentes de saúde e os usuários parece ter favorecido a busca destes pelo tratamento. Essa boa relação pode ter sido construída desde o período em que houve o surto de malária na cidade e intensificada a partir das ações de vigilância preconizadas pelo programa nacional de controle da malária.

A interação entre o profissional de saúde e o usuário tem sido relatada na literatura como elemento fundamental para que este último cuide apropriadamente de sua saúde. Profissionais têm reconhecido a importância da comunicação efetiva e de boa qualidade com os usuários, permitindo a eles o acesso ao conhecimento sobre os problemas de saúde e os tratamentos, bem como a possibilidade de sanar suas dúvidas. ${ }^{25}$

Constatada a presença do parasita no sangue e determinado o tipo de malária contraída, o usuário recebia instruções verbais e um envelope com os medicamentos específicos e as instruções escritas e com imagens pictóricas de como tomar os medicamentos corretamente [O agente de saúde] colocou dentro de papelzinho escrito, depois explicou ainda. (E8); Aí eles já marcam certinho, conversa com a gente como é que é. Já vem marcado certinho (E7).

Entende-se que as instruções sobre a ingestão correta dos medicamentos pode ter tido um efeito significativo sobre o comportamento dos usuários pesquisados em relação ao tratamento. A adesão dos pacientes melhora notavelmente quando às instruções verbais do pessoal de saúde se agrega a entrega de instruções por escrito em forma de desenhos ou diagrama ${ }^{(26)}$. Estudos têm comprovado a eficácia dessa estratégia e apontado para a necessidade de investimento na mesma..$^{21,27-28}$

\section{Adesão ao tratamento e motivos para aderir}

Embora tenham buscado o tratamento antimalárico e recebido as informações e instruções de como proceder em relação ao mesmo, nem todos os usuários seguiram-no como prescrito. Quando questionados se tinham seguido o tratamento conforme recomendado pelos agentes de saúde, os 14 usuários que buscaram o tratamento preconizado pelo MS para tratar a malária declararam que sim.

Entretanto, quatro deles relataram ter se esquecido de tomar a medicação no horário prescrito ou que a malária voltou $P$ - E o senhor conseguiu tomar esses medicamentos?E -Consegui. P - Todos eles? E - Todos. P - Chegou a falhar algum? E - Não, às vezes atrasava no horário, mas depois eu tomava. Não deixava de tomar não (E4).

Esquecer ou deixar de tomar os medicamentos nos dias, horários e quantidades determinados tem sido apontado por autores como um dos fatores contribuintes à não-adesão ao tratamento. ${ }^{26,29}$ Entretanto, a repercussão dessas ações é diferente a depender do tipo de enfermidade que a pessoa tem.

Em tratamentos de longa duração, como os das doenças crônicas, esquecer ou deixar de tomar os medicamentos pode ser significativo em termos de adesão apenas se for um comportamento frequente.

No caso do tratamento antimalárico, no entanto, é fundamental que os medicamentos sejam ingeridos nos dias, horários e quantidades determinados, uma vez que a não observância disso pode levar à resistência aos mesmos e também a recaídas que, por sua vez, favorecem a manutenção da pessoa como foco de infecção e agente de transmissão. ${ }^{14,17}$

Assim, pelas evidências encontradas nos relatos daqueles quatro usuários, considerou-se que provavelmente eles não aderiram ao tratamento.

Por outro lado, considerando-se a definição de adesão ao tratamento deste estudo, dez dos usuários provavelmente aderiram, pois ao longo de seus relatos não se evidenciaram inconsistências ou incoerências em relação ao seguimento do tratamento prescrito $P$ - E quando começou a trabalhar conseguiu tomar os remédios na hora certa? E - Sim. P - Como o senhor fazia? E - Levava relógio prá roça e o remédio junto (E3).

Nos relatos desses usuários foi possível identificar alguns motivos para seguirem as recomendações dos agentes de saúde em relação ao tratamento da malária. Tais motivos parecem ter origem na vivência do usuário com a doença.

O primeiro deles foi a presença dos sinais e sintomas que, segundo eles, eram ruins e traziam muito sofrimento. A malária é terrível, judia da gente (E16); Então, seguiam o tratamento porque senão a malária não sarava e a gente sofria o tempo todo (E3). 
Outro motivo foi o desejo de melhorar e ficar curado. [Eu tomava os medicamentos] porque eu queria melhorar (E14); Eu tomei o remédio direitinho para me ver livre da doença o quanto antes (E16).

Os usuários também relataram que optaram por tomar os antimaláricos por terem medo de piorar e voltar a apresentar os sinais e sintomas da doença. [...] porque eu ia ficar ruim de novo (E6); - Continuei tomando porque se não tratasse direitinho ela voltava (E11). Resultado similar foi encontrado num estudo ${ }^{30}$ sobre adesão ao tratamento da malária no Peru.

Os motivos mencionados pelos usuários fazem sentido na medida em que se sabe que toda pessoa, ao ficar doente, tem, ao mesmo tempo, medo de piorar e vontade de melhorar o mais rápido possível, seja para se ver livre da doença ou para voltar a trabalhar. Em uma pesquisa, os participantes tinham vontade de se recuperar da malária para continuar seu trabalho na agricultura e minimizar suas perdas. ${ }^{31}$

Por outro lado, estudos têm destacado a melhora dos sinais e sintomas como uma das razões para as pessoas abandonarem o tratamento. ${ }^{29-30}$ Tão logo se sentem melhor, as pessoas deixam de tomar os medicamentos e de dar prosseguimento ao tratamento. No caso da malária, isso leva a recaídas, recrudescimento e favorece a resistência aos antimaláricos. ${ }^{14,17}$ Esse pode ter sido o caso de alguns dos usuários que relataram que a malária voltou. [...] antes de sarar direito de uma, eu já pegava outra de novo (E7).

Por fim, o quarto motivo baseou-se na eficácia do tratamento percebida pelos usuários. Segundo eles, os medicamentos eram eficazes no combate à malária. Porque estava fazendo efeito, não estava fazendo mal, aí não precisava parar (E9); Se estava fazendo bem, porque parar? (E10) e se constituíam na única forma de cura definitiva da doença. Tomava tudo porque senão não matava a malária (E13); Porque daí não sarava a malária (E8). Nesta pesquisa, portanto, presume-se que a aceitação do esquema terapêutico antimalárico por parte dos usuários foi boa, resultado que se assemelha aos encontrados em outros estudos ${ }^{18,19-30}$ embora neste último, os participantes da pesquisa considerassem os antimaláricos como tratamento complementar às plantas medicinais.

\section{CONCLUSÃO E CONSIDERAÇÕES}

Este estudo descreveu a adesão e as reações dos usuários dos serviços públicos de saúde do município de Juruena em relação ao tratamento antimalárico e revelou que a maioria deles, tão logo começavam a apresentar os sinais e sintomas da malária, buscavam o serviço de saúde para obter o diagnóstico e o tratamento apropriado. Em seus relatos foi possível apreender que alguns fatores podem ter contribuído para isso: o conhecimento deles sobre a doença, a forma de transmissão e o tratamento; o acesso ao serviço público de saúde, ao diagnóstico e ao tratamento gratuitos; e o bom relacionamento entre os agentes de saúde e os usuários.

A principal estratégia para garantir bons e sustentáveis resultados é a integração das ações de luta contra a malária no sistema permanente, fortificado e atuante de saúde local, sendo uma referência clara e próxima para a população. ${ }^{3}$

Ao que parece, pelos depoimentos dos usuários, as ações de controle da malária desenvolvidas no município estão atendendo às recomendações dos programas do governo, no que diz respeito ao fornecimento de informações sobre a doença, seus mecanismos de transmissão e de controle e a importância do diagnóstico e tratamento precoces.

Neste sentido, considera-se que há necessidade de se manter essas ações, entretanto é necessário incrementá-las, pois a educação em saúde implica muito mais do que fornecer informações. O trabalho educativo deve ser feito de modo a problematizar e criar espaços de diálogo e participação entre as pessoas envolvidas, compartilhando novos saberes e práticas que respeitem as peculiaridades pessoais, sociais, econômicas, culturais e ambientais que levam a formas específicas de comportamentos das pessoas, famílias e comunidade, fortalecendo a capacidade individual e coletiva dos usuários de lidar com os múltiplos fatores que contribuem para seu adoecer.

Deve ainda, superar a relação autoritária e normatizadora muitas vezes presente entre o profissional de saúde e o usuário, entre o serviço de saúde e a população. Neste contexto, o papel dos profissionais de saúde é o de reconhecer a educação em saúde como uma tarefa inerente ao seu trabalho e que, por isso devem se dedicar a desenvolvê-la no cotidiano de suas práticas.

De outro modo, este estudo também revelou que, embora a maioria dos usuários tenha buscado o tratamento antimalárico oficial, ainda há pessoas que aderem parcialmente a ele e outras que buscam tratamentos alternativos.

Esses achados devem ser motivo de preocupação por parte dos profissionais de saúde, uma 
vez que os usuários que não buscam o tratamento ou os que não aderem constituem fonte de infecção e transmissão da doença, tornando-se um obstáculo para o controle efetivo da malária. Esses resultados ainda sustentam a necessidade de mais investimento em educação em saúde, incremento nas ações de vigilância em saúde e melhora do acesso aos serviços de saúde.

Todo o investimento dos governos para o controle da malária, incluindo a disponibilização das drogas antimaláricas, poderá ser perdido se não forem elaboradas e implementadas estratégias que visem aumentar a adesão do paciente portador de malária ao tratamento.

Tais ações têm implicações importantes para a saúde das pessoas na medida em que pode capacitar a população para lidar tanto com a malária quanto com outros problemas de saúde de maneira apropriada e eficaz. Além disso, pode contribuir para a redução das perdas sociais e econômicas que as doenças trazem para as pessoas, as famílias e a comunidade.

Embora esta pesquisa tenha trazido informações relevantes sobre as reações das pessoas em relação ao tratamento antimalárico, é necessário que mais estudos sejam desenvolvidos em outros cenários, com outras populações e outras abordagens a fim de que se amplie o conhecimento sobre a forma como as pessoas lidam com seus problemas de saúde, principalmente nos casos em que há focos de transmissão da doença. Em consequência, os profissionais de saúde, agências de governo municipal e estadual da saúde, órgãos não-governamentais e a comunidade poderão repensar e redefinir suas estratégias de ação, a partir de dados vindos da realidade e se adequar a elas.

\section{REFERÊNCIAS}

1. Butcher CA. Malaria: a parasitic disease. AAOHN Journal 2004 Jul; 52(7):302-9.

2. Tauil PL. Perspectivas de controle de doenças transmitidas por vetores no Brasil. Rev Soc Bras Med Trop. 2006 Mai-Jun; 39(3):275-7.

3. Silveira AC; Rezende DF. Avaliação da estratégia global de controle integrado da malária no Brasil. Brasília: Organização Pan-Americana da Saúde 2001.

4. Rede Interagencial de Informações para Saúde. Índice parasitário anual [página da Internet]. Brasília: RIPSA; 2004 [acesso 2007 Mar 17]. Disponível em: http:/ / www.ripsa.org.br/fichasIDB/record. php?node=D.4\&lang=pt.
5. Ministério da Saúde (BR). Situação epidemiológica da malária no Brasil: ano de 2006. Brasília: Secretaria de Vigilância em Saúde; Ministério da Saúde; 2007.

6. Duarte EC, Gyorkos TW. Self-reported compliance with last malaria treatment and occurrence of malaria during follow-up in a Brazilian Amazon population. Trop Med Int Health 2003 Jun; 8(6):518-24.

7. Ministério da Saúde (BR). Programa Nacional de prevenção e controle da malária - PNCM. Brasília: Ministério da Saúde; 2003.

8. Yeung S, White NJ. How do patients use antimalarial drug? A review of the evidence. Trop Med Int Health 2005 Feb; 10(2):121-38.

9. Ministério da Saúde (BR), Secretaria de Vigilância em Saúde, Secretaria de Atenção à Saúde. Política nacional de promoção da saúde. Brasília (DF): MS; 2006.

10. Polit DF, Beck CT, Hungler BP. Fundamentos de pesquisa em enfermagem: métodos, avaliação e utilização. $5^{\mathrm{a}}$ ed. Porto Alegre (RS): Artmed; 2004.

11. Instituto Brasileiro de Geografia e Estatística. Cidades, estatísticas populacionais. [página da internet]. Brasília (DF): IBGE. [acesso 2008 Abr 4]. Disponível em: http:// www.ibge.gov.br/ cidadesat/topwindow.htm?1.

12. Prefeitura Municipal de Juruena. Dados econômicos. [página da internet]. Juruena (MT): Prefeitura Municipal de Juruena; 2007 [acesso 2008 Abr 5]. Disponível em: http:/ / www.pmjuruena.com.br/ index.php?exibir $=$ secoes $\& I D=13$

13. Secretaria Estadual de Saúde de Mato Grosso. Boletim epidemiológico da malária. Cuiabá (MT): SES; 2005.

14. Ministério da Saúde (BR). Secretaria de vigilância em saúde. Ações de controle da malária: manual para profissionais de saúde na atenção básica. Brasília: Ministério da Saúde; 2006.

15. Ministério da Saúde (BR). Coordenação Nacional de doenças sexualmente transmissíveis e Aids do Ministério da Saúde. Aderência ao tratamento com anti-retrovirais em serviços públicos no estado de São Paulo. [internet]. 2006 [acesso 2007 Jan 17]. Disponível em: http:/ / www.aids.gov.br/data/documents/ storedDocuments / \% 7BB8EF5DAF-23AE-4891AD36-1903553A3174\% 7D / \% 7BF A7B43027CF3-4481-A119-E66B3748C76B\%7D/S\%E9rie_ Avalia\%E7\%E3o_1.pdf.

16. McCombie SC. Treatment seeking for malaria: a review of recent research. Soc Sci Med. 1996 Sep; 43(6):933-45.

17. World Health Organization. WHO guidelines for the treatment of malaria. Geneva (CH): WHO; 2006.

18. Oberländer L, Elverdan B. Malaria in the United Republic of Tanzania: cultural considerations and health-seeking behaviour. Bull World Health Organ. 2000; 78(11):1352-7. 
19. Vigneron M, Deparis X, Deharo E, Bourdy G. Antimalarial remedies in French Guiana: a knowledge attitudes and practices study. J Ethnopharmacol. 2005 Apr; 98(3):351-60.

20. Kaona FAD, Tuba M. A qualitative study to identify community structures for management of severe malaria: a basis for introducing rectal artesunate in the under five years children in Nakonde District of Zambia. BMC Public Health [online] 2005 Mar [cited 2006 Dec 20]; 5:28. Available from: http://www. biomedcentral.com/1471-2458/5/28.

21. Dike N, Onwujekwea O, Ojukwuc J, Ikemed A, Uzochukwue B, Shu E. Influence of education and knowledge on perceptions and practices to control malaria in Southeast Nigeria. Soc Sci Med. 2006 Jul; 63(1):103-6.

22. Czeresnia D. O conceito de saúde e a diferença entre prevenção e promoção. In: Czeresnia D, Freitas CM. Promoção da saúde: conceitos, reflexões, tendências. Rio de Janeiro (RJ): Fiocruz; 2003. P.39-53.

23. Fogg C, Bajunirwe F, Piola P, Biraro S, Checchi F, Kiguli J. et al. Adherence to a six-dose regimen of artemether-lumefantrine for treatment of uncomplicated Plasmodium falciparum malaria in Uganda. Am J Trop Med Hyg. 2004 Nov; 71(5):525-30.

24. Simsek Z, Kurcer MA. Malaria: knowledge and behaviour in an endemic rural area of Turkey. Public Health 2005 Mar; 119(3):202-8.
25. Clancy C. How do we involve patients in their own healthcare decisions? [vídeo] Med Gen Med. 2007 [acesso 2007 Dec 15]; 9(4):46. Avaliable from: http:// www.medscape.com/viewarticle/565951.

26. Yépez MC, Zambrano D, Carrasco F, Yépez RF. Factores asociados con el incumplimiento del tratamiento antipalúdico en pacientes ecuatorianos. Rev Cubana Med Trop. 2000 May-Aug; 52(2):81-9.

27. Okonkwo PO, Akpala CO, Okafor HU, Mbah AU, Nwaiwu O. Compliance to correct dose of chloroquine in uncomplicated malaria correlates with improvement in the condition of rural Nigerian children. Trans R Soc Trop Med Hyg. 2001May-Jun; 95(3): 320-24.

28. Kools M, van de Wiel MW, Ruiter RA, Kok G. Pictures and text in instructions for medical devices: effects on recall and actual performance. Patient Edu Couns. 2006 Dec; 64(1-3):104-11.

29. Reiners AAO, Azevedo RCS, Vieira MA, Arruda ALG. Produção bibliográfica sobre adesão/nãoadesão de pessoas ao tratamento de saúde. Ciênc Saúde Coletiva. 2008; 13(Sup2):2299-306.

30. Llanos-Zavalaga F, Cotrina A, Campana P. Factores asociados a la adherencia al tratamiento de malaria en Piura y Tumbes -Perú. Rev Med Exp 2001; 18(3-4):63-70.

31. Nyamongo IN. Health care switching behaviour of malaria patients in a Kenyan rural community. Soc Sci Med 2002 Feb; 54(3):377-86. 